\title{
The E3 ubiquitin ligase TRIM62 and inflammation- induced skeletal muscle atrophy
}

\author{
Franziska Schmidt ${ }^{1 \dagger}$, Melanie Kny ${ }^{1 \dagger}$, Xiaoxi Zhu' ${ }^{1}$, Tobias Wollersheim²${ }^{2}$, Kathleen Persicke ${ }^{1}$, Claudia Langhans ${ }^{1}$, \\ Doerte Lodka', Christian Kleber ${ }^{3}$, Steffen Weber-Carstens ${ }^{2}$ and Jens Fielitz ${ }^{1,4^{*}}$
}

\begin{abstract}
Introduction: ICU-acquired weakness (ICUAW) complicates the disease course of critically ill patients. Inflammation and acute-phase response occur directly within myocytes and contribute to ICUAW. We observed that tripartite motif-containing 62 (TRIM62), an E3 ubiquitin ligase and modifier of inflammation, is increased in the skeletal muscle of ICUAW patients. We investigated the regulation and function of muscular TRIM62 in critical illness.

Methods: Twenty-six critically ill patients with Sequential Organ Failure Assessment scores $\geq 8$ underwent two skeletal muscle biopsies from the vastus lateralis at median days 5 and 15 in the ICU. Four patients undergoing elective orthopedic surgery served as controls. TRIM62 expression and protein content were analyzed in these biopsies. The kinetics of Trim62, Atrogin1 and MuRF1 expression were determined in the gastrocnemius/plantaris and tibialis anterior muscles from mouse models of inflammation-, denervation- and starvation-induced muscle atrophy to differentiate between these contributors to ICUAW. Cultured myocytes were used for mechanistic analyses.

Results: TRIM62 expression and protein content were increased early and remained elevated in muscles from critically ill patients. In all three animal models, muscular Trim62 expression was early and continuously increased. Trim62 was expressed in myocytes, and its overexpression activated the atrophy-inducing activator protein 1 signal transduction pathway. Knockdown of Trim62 by small interfering RNA inhibited lipopolysaccharide-induced interleukin 6 expression.
\end{abstract}

Conclusions: TRIM62 is activated in the muscles of critically ill patients. It could play a role in the pathogenesis of ICUAW by activating and maintaining inflammation in myocytes.

Trial registration: Current Controlled Trials ID: ISRCTN77569430 (registered 13 February 2008)

\section{Introduction}

ICU-acquired weakness (ICUAW) is a devastating complication of critical illness characterized by loss of muscle mass [1], preferential atrophy of fast-twitch myofibers and weakness [2-4]. Affected patients face a prolonged hospital stay and mechanical ventilation, increased hospital mortality and chronic physical disability $[5,6]$. The pathophysiology of ICUAW is poorly understood [7]. However, we [8] and others [1] have shown that dysbalanced muscular protein homeostasis due to increased protein degradation and reduced protein synthesis occurs in muscle of critically ill

\footnotetext{
*Correspondence: jens.fielitz@charite.de

${ }^{\dagger}$ Equal contributors

'Experimental and Clinical Research Center (ECRC), a Cooperation between Max-Delbrück-Centrum and Charité-Universitätsmedizin Berlin, Campus Buch, Lindenberger Weg 80, 13125 Berlin, Germany

${ }^{4}$ Cardiology, Charité-Universitätsmedizin Berlin, Campus Virchow,

Augustenburger Platz 1, 13353 Berlin, Germany

Full list of author information is available at the end of the article
}

patients and may contribute to ICUAW $[1,2,8,9]$. Breakdown of muscular proteins such as myosin heavy chain $(\mathrm{MyHC})$ is mediated by the ubiquitin-proteasome system (UPS) [10], which is activated in muscle of critically ill patients $[1,8,11]$ and involves the F-box adaptor protein FBXO32/Atrogin1 [12] and the E3 ubiquitin ligase muscle RING (really interesting new gene) finger-containing protein 1 (MuRF1). Atrogin1 and MuRF1 are rapidly and transiently increased in the skeletal muscle of critically ill patients [8]. However, muscle atrophy and regulation of Atrogin1 and MuRF1 expression are not synchronized, because atrophy occurs later in the disease process, when Atrogin1 and MuRF1 have already returned to baseline [8]. This discrepancy argues for additional continuously activated atrophy pathways. Chronic and persistent inflammation and acute-phase response directly occurring in the skeletal muscle of critically ill patients might be one of these

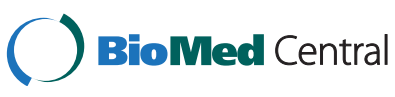

(c) 2014 Schmidt et al.; licensee BioMed Central Ltd. This is an Open Access article distributed under the terms of the Creative Commons Attribution License (http://creativecommons.org/licenses/by/4.0), which permits unrestricted use, distribution, and reproduction in any medium, provided the original work is properly credited. The Creative Commons Public Domain Dedication waiver (http://creativecommons.org/publicdomain/zero/1.0/) applies to the data made available in this article unless otherwise stated. 
mechanisms [13]. Recently, we have shown that interleukin 6 (IL-6) and the acute-phase response proteins serum amyloid A1 (SAA1) and SAA4 are continuously elevated in the muscle of critically ill patients [13]. Both IL-6 [14,15] and SAA1 $[16,17]$ are known to induce atrophy by increasing protein degradation in the skeletal muscle of both patients and rodents. We performed a gene expression array and found the modifier of inflammation tripartite motif-containing 62 (TRIM62) to be increased in the muscle of critically ill patients [13]. TRIM62 belongs to the family of RING finger E3 ubiquitin ligases $[18,19]$ and was identified as a dominant regulator of acinar morphogenesis in the mammary gland [20]. Strong evidence exists that TRIM62 plays a role in Toll-like receptor 4 (TLR4) signaling. More specifically, TRIM62 activates the Toll/interleukin 1 receptor domain-containing adapter inducing interferon $\beta$ (TRIF) branch of the TLR4 signaling pathway, leading to increased activity of the activator protein 1 (AP-1) transcription factor in primary macrophages [21]. Because AP-1 signaling is essential for denervation-induced atrophy [22], we hypothesized that TRIM62-mediated activation of AP-1 signaling in myocytes contributes to inflammation-induced atrophy in critically ill patients. To specifically focus on early time points of muscle atrophy and to differentiate between the major contributors of ICUAW, we relied on three mouse atrophy models described elsewhere: cecal ligation and puncture (CLP) mimicking sepsis, denervation-induced atrophy and food deprivation [13]. These models were used to compare the kinetics of Trim62 with Atrogin1 and $M u R F 1$ gene expression in muscle. Cultured myocytes and reporter gene assays were used for mechanistic analyses.

\section{Material and methods Patients}

The institutional review board of the Charité approved the study, and written informed consent was obtained from the patient or the patient's legal proxy (Charité EA2/061/ 06). We recently reported clinical data and molecular analyses in the biopsy specimens of the same patients $[2,8,13,23]$. We specifically included patients at high risk of developing ICU-acquired muscle wasting and weakness [24]. Open muscle biopsies from the vastus lateralis were performed at median day 5 in 26 ICU patients (early time point). Of these 26 patients, 14 remained at least to median day 15 in the ICU (late time point), when a second biopsy specimen from the vastus lateralis was obtained. Four age- and gender-matched patients undergoing elective orthopedic surgery, otherwise healthy, permitted a biopsy from the vastus lateralis at the time point of elective surgery. For further details, refer to Additional file 1.

\section{Animal models of muscle atrophy}

To focus on early time points of muscle atrophy, we relied on three mouse models described elsewhere: CLP surgery that mimics sepsis, denervation-induced atrophy and food deprivation $[13,25,26]$. All animal procedures were performed in accordance with the guidelines of the Max-Delbrück Center for Molecular Medicine and the Charité-Universitätsmedizin Berlin and were approved by the Landesamt für Gesundheit und Soziales (LaGeSo, Berlin, Germany) for the use of laboratory animals (permit number G 0129/12). They followed the principles of laboratory animal care set forth by the National Institutes of Health (NIH) in the Guide for the Care and Use of Laboratory Animals (NIH Publication 86-23, revised 1985), as well as the current version of the German Law on the Protection of Animals. Briefly, 6- to 8-week-old male C57BL/6 $\mathrm{N}$ mice were used for all experiments. CLP surgery was performed to induce polymicrobial sepsis according to a published protocol [25] and as recently reported [13]. Sham mice were treated identically, except for the ligation and puncture of the cecum. CLP $(n=4$ or 5$)$ and sham-treated $(n=4$ or 5$)$ mice were sacrificed 24 hours, 48 hours, 72 hours or 96 hours after surgery. Neurogenic atrophy was induced by dissection of the left sciatic nerve (denervation). The sciatic nerve of the right leg was cut, and a 3-mm piece was excised (denervated). The right leg remained innervated and was used as the control (innervated). Mice were sacrificed at baseline or 7 days, 14 days or 21 days postsurgery $(n=6$ each). Food deprivation was performed for 0 hours (control), 24 hours or 48 hours ( $n=6$ each).

For detailed information about animal experiments, quantitative RT-PCR (qRT-PCR), immunohistology, immunoblotting, myoblast culture and immunocytology, small interfering RNA (siRNA) transfection and luciferase reporter assay, please refer to Additional files 1 .

\section{Statistical tests}

A two-sided Mann-Whitney $U$ test was used to determine statistical differences. Data shown are mean \pm SEM. Statistical tests were calculated using GraphPad Prism software (GraphPad Software, La Jolla, CA, USA). Box plots showing medians with 25th and 75th percentiles were made using GraphPad Prism 5. $P<0.05$ was considered statistically significant.

\section{Results}

TRIM62 mRNA was upregulated in muscle of critically ill patients

The study protocol (Additional file 2: Figure S1), data on patients' characteristics (Additional file 1: Table S1) and treatment (Additional file 1: Table S2) are provided. The data from our microarray have been deposited in the National Center for Biotechnology Information Gene Expression Omnibus (GEO) database [GEO:GSE53702] (http://www.ncbi.nlm.nih.gov/ geo/query/acc.cgi?acc=GSE53702). We found a 3.9-fold 
upregulation of TRIM62 [13]. We performed qRT-PCR in a subset of our recently published ICU patients [8] at the early (day $5, n=26$ ) and late (day $15, n=14$ ) time points and orthopedic controls $(n=4)$ to confirm these data. Continuous upregulation of muscular TRIM62 mRNA was confirmed for both time points of critical illness $(P<0.01)$ (Figure 1A). Immunoblot analysis showed that muscular TRIM62 protein was increased at the early time point and remained elevated until the late time point in critically ill patients (Figure 1B). In contrast, Atrogin1 and MuRF1 expression was significantly increased in the early and returned to control levels in the late biopsy specimens in these patients as recently reported [8].

\section{Trim62 was persistently elevated during skeletal muscle atrophy in mice}

Because Trim62 has been shown to be involved in inflammatory response of immune cells [21], we reasoned that Trim62 could play a role in the development of ICUAW. We used three standard animal models of muscle atrophy-CLP $[13,25]$, denervation [26] and food deprivation - to investigate regulation of Trim62 and to compare it with the expression of the standard atrophy markers Atrogin1 and MuRF1.

\section{Muscular Trim62 expression was increased during inflammation-induced skeletal muscle atrophy in mice} Inflammation-induced muscle atrophy was induced by the CLP method of polymicrobial sepsis as previously described [13,25]. Recently, we reported that increased expression of $\mathrm{Il}-6$ and Saa1 in muscles of CLP mice might contribute to inflammation-induced atrophy [13,25]. In the present study, general inflammation was confirmed by an early and persistent increase in gene expression of the inflammatory cytokines $\mathrm{Il}-6$ and tumor necrosis factor $\alpha$ $(\operatorname{Tnf}-\alpha)$, as well as of the acute-phase protein Saa1, in the liver of CLP mice (Additional file 3: Figure S2). Septic mice showed a significant decrease in body weight as soon as 24 hours after surgery. CLP mice continued their weight loss, which reached its maximum after 96 hours at a reduction of $19 \%$ of body weight $(P<0.05)$ (Additional file 4: Figure S3A). A significant decrease in muscle weights was found after 72 hours and 96 hours of sepsis, indicative of muscular atrophy (Figure 2A). Using qRT-PCR, we found a significant increase in Atrogin1 and MuRF1 expression after 24 hours of sepsis in gastrocnemius/plantaris and tibialis anterior muscles. Atrogin1 and MuRF1 expression followed a time course with early induction and a subsequent decrease (Figures $2 \mathrm{~B}$ and $2 \mathrm{C}$ ). Whereas MuRF1 expression was highest at the 24-hour time point, the induction of Atrogin1 peaked at 48 hours of sepsis (Figures $2 \mathrm{~B}$ and 2C). Immunoblot analysis confirmed that muscular MuRF1 protein content went in parallel with its expression, showing an early induction and a subsequent decrease in gastrocnemius/plantaris and tibialis anterior muscles of CLP mice (Figure 2D).

Muscular Trim62 expression was measured by qRTPCR. It was significantly increased in gastrocnemius/ plantaris and tibialis anterior muscles at 48 hours and 72 hours after CLP surgery. Throughout the experiment, Trim62 expression levels were persistently elevated in both muscle types (Figure 3A).

Muscular Trim62 expression was increased in denervation-induced skeletal muscle atrophy in mice We investigated if Trim62 was regulated in neurogenic muscle atrophy. A progressive loss of muscle weight was

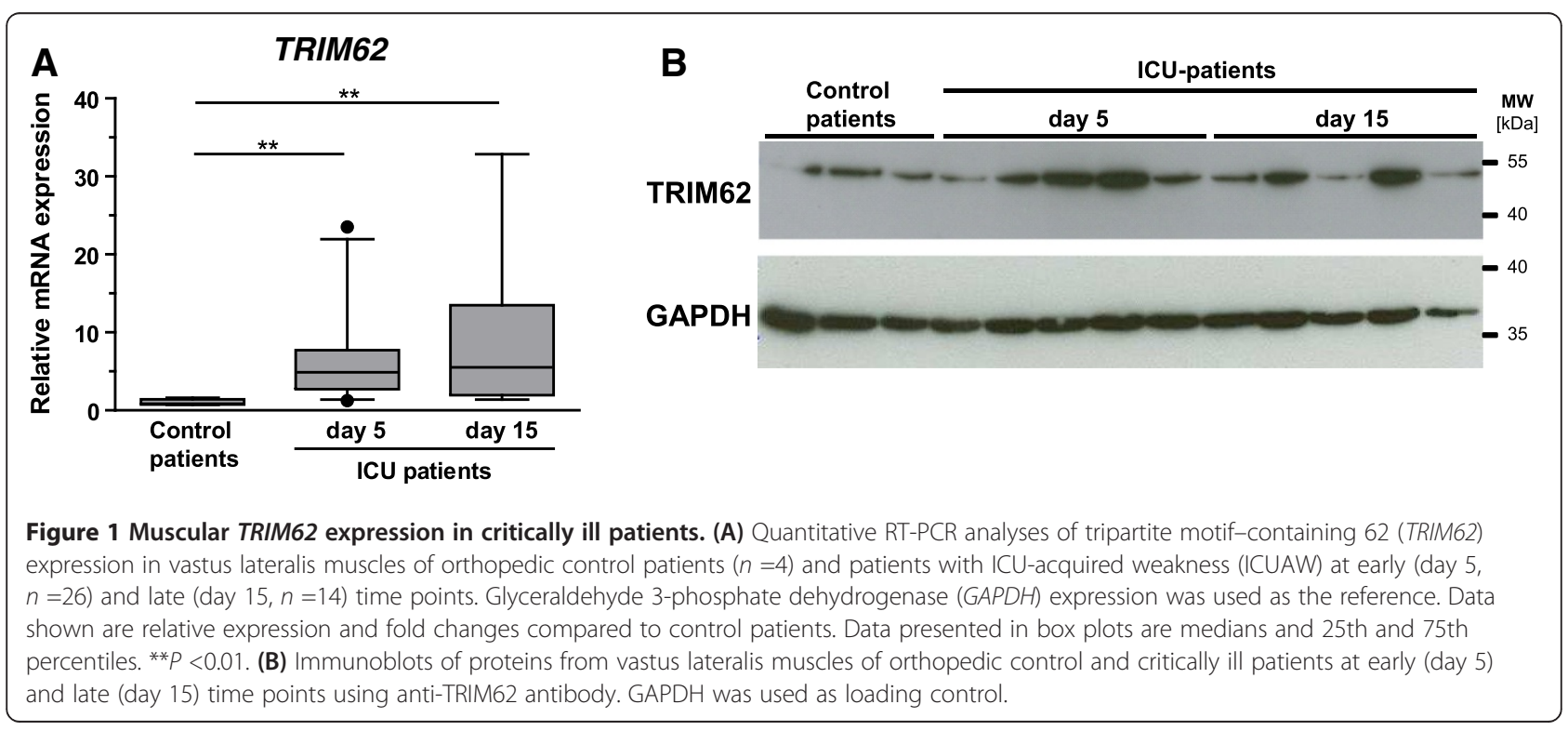


A

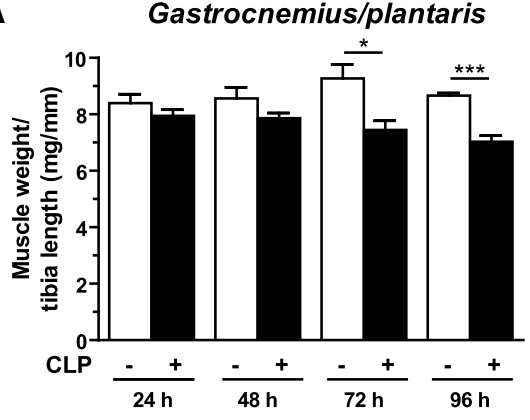

B

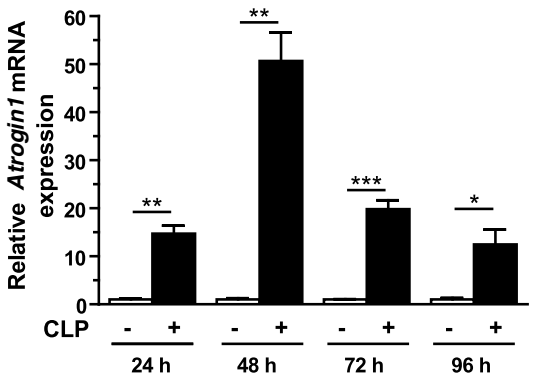

C
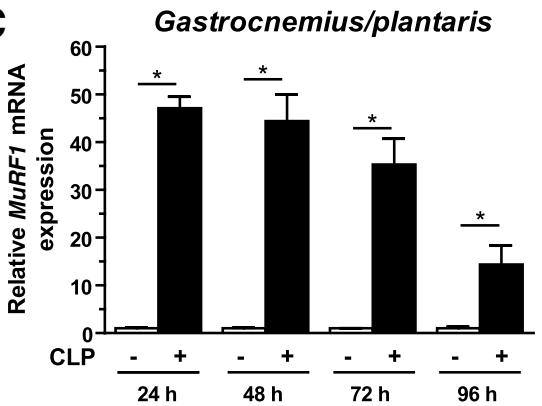

D

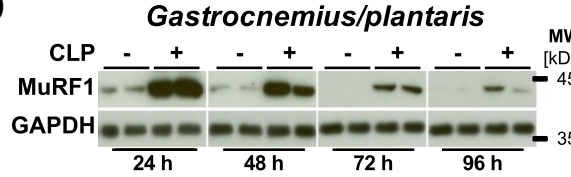

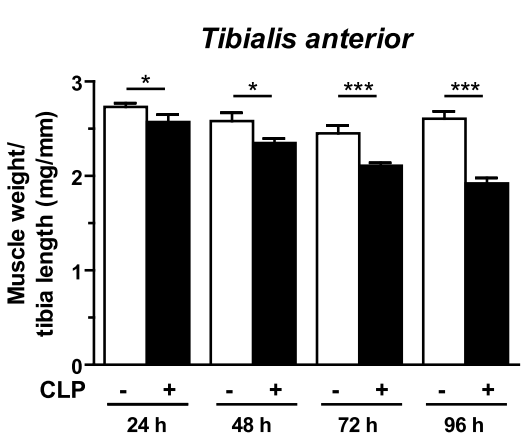

Tibialis anterior
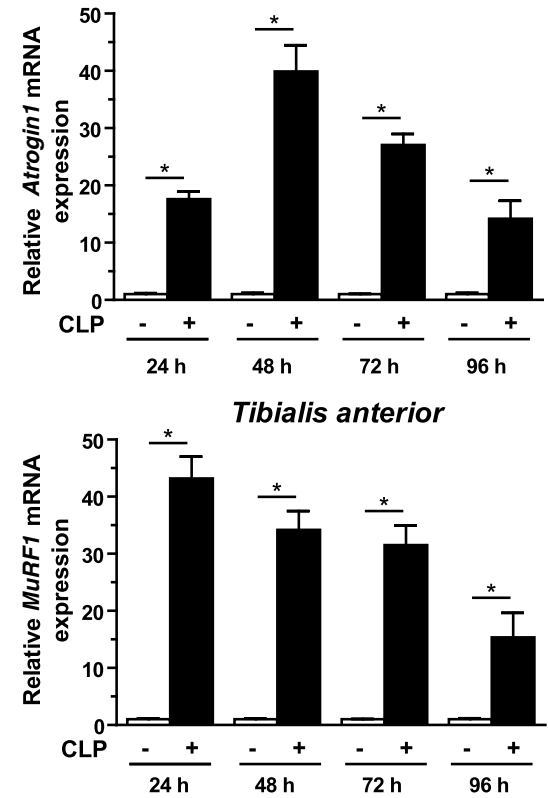

Tibialis anterior

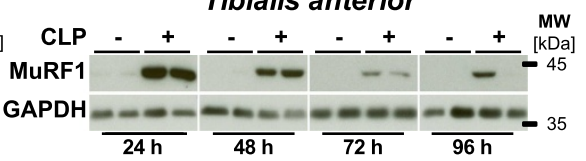

Figure 2 Inflammation leads to skeletal muscle atrophy in vivo. Six- to eight-week-old male C57BL/6 N mice were subjected to sham operations (sham, $n=5$ ) or cecal ligation and puncture (CLP) surgery $(n=5)$, as indicated, to induce polymicrobial sepsis. (A) Weights of the gastrocnemius/plantaris and tibialis anterior muscles were determined after 24 hours, 48 hours, 72 hours or 96 hours and normalized to tibia length. Data are presented as mean \pm SEM. ${ }^{* *} P<0.001,{ }^{*} P<0.05$. Quantitative RT-PCR analyses of Atrogin1 (B) and MURF1 (C) expression, and immunoblotting of proteins using anti-MuRF1 antibody (D), in gastrocnemius/plantaris and tibialis anterior muscles at 24 hours, 48 hours, 72 hours or 96 hours after surgery, as indicated. Glyceraldehyde 3-phosphate dehydrogenase (Gapdh) expression and protein content were used as reference values, and data shown are relative changes. Data are presented as mean \pm SEM. ${ }^{* *} P<0.001,{ }^{* *} P<0.01,{ }^{*} P<0.05$. MuRF1, Muscle RING (really interesting new gene) finger-containing protein 1.

observed for gastrocnemius/plantaris and tibialis anterior muscles. Weights of gastrocnemius/plantaris and tibialis anterior muscles were significantly decreased after 7 days of denervation (Figure 4A). A continuous decrease in muscle mass was observed until 21 days of denervation, reaching $54 \%$ and $49 \%$ ( $P<0.01$ for both) of muscle weight for gastrocnemius/plantaris and tibialis anterior muscles, respectively (Figure 4A). Atrogin1 (Figure 4B) and MuRF1 (Figure 4C) expression was significantly increased at both time points in gastrocnemius/plantaris and tibialis anterior muscles. More specifically, Atrogin1 and MuRF1 expression was highest after 7 days of denervation. Atrogin1 expression was 8.3- and 4.2-fold upregulated in gastrocnemius/plantaris and tibialis anterior muscles, respectively, after 7 days of denervation ( $P<0.01$ for both) (Figure 4B). At the same time point, $M u R F 1$ expression was increased four- and threefold in gastrocnemius/plantaris and tibialis anterior muscles, 

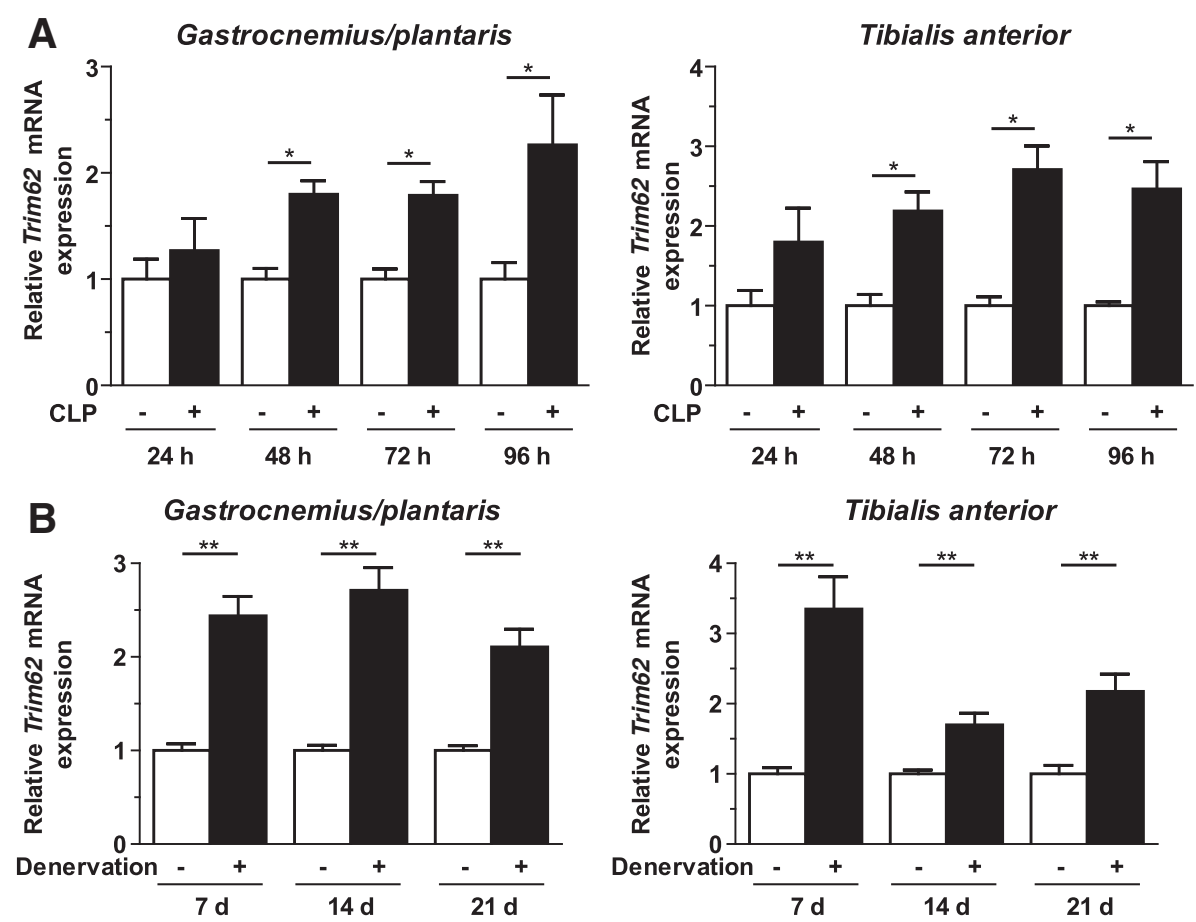

Tibialis anterior

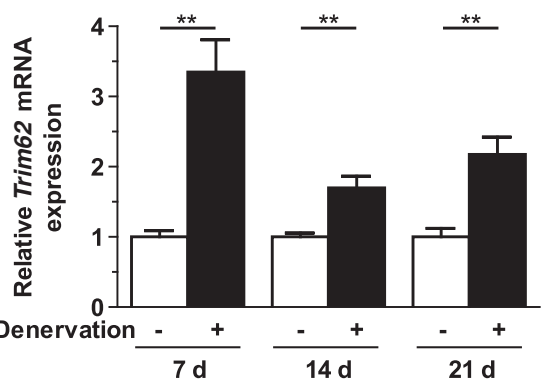

C Gastrocnemius/plantaris
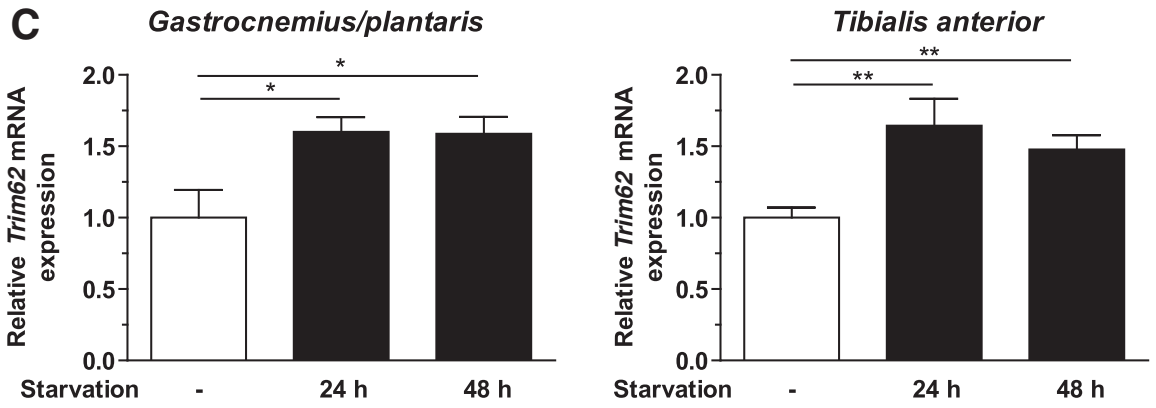

Figure 3 Trim62 expression is increased during inflammation-, denervation- and starvation-induced muscle atrophy. (A) Quantitative RT-PCR (qRT-PCR) analyses of Trim62 expression in gastrocnemius/plantaris and tibialis anterior muscles at 24 hours, 48 hours, 72 hours and 96 hours after surgery, as indicated. CLP, Cecal ligation and puncture surgery. (B) qRT-PCR analyses of Trim62 expression in gastrocnemius/plantaris and tibialis anterior muscles 7 days, 14 days and 21 days after denervation or sham surgery. (C) qRT-PCR analyses of Trim62 expression in gastrocnemius/ plantaris and tibialis anterior muscles of control mice $(-, n=6)$ and mice deprived of food for 24 hours $(n=6)$ or 48 hours $(n=6)$, as indicated. Glyceraldehyde 3-phosphate dehydrogenase (Gapdh) expression was used as the reference in all assays. Data shown are fold changes of expression in sham-operated and untreated mice. Data are presented as mean \pm SEM. ${ }^{*} P<0.01,{ }^{*} P<0.05$.

respectively ( $P<0.01$ for both) (Figure $4 \mathrm{C}$ ). The MuRF1 protein content showed a comparable time course during denervation in gastrocnemius/plantaris and tibialis anterior muscles (Figure 4D).

Trim62 expression was also significantly increased at both time points in denervated gastrocnemius/plantaris and tibialis anterior muscles (Figure 3B). In gastrocnemius/plantaris muscles, a 2.4-fold upregulation of Trim62 was measured 7 days after denervation, and it remained at this level at 14 days and 21 days $(P<0.01$ for all). In tibialis anterior muscle, Trim62 expression reached its maximum at 7 days (3.3-fold induction) and decreased thereafter (1.7-fold after
14 days and 2.2-fold after 21 days $)(P<0.01$ for both) (Figure 3B).

\section{Starvation-induced atrophy was accompanied by} increased Trim62 expression in mouse skeletal muscle To investigate the impact of starvation on muscle atrophy, mice were food-deprived for 24 hours and 48 hours. Food deprivation led to decreases in body weight (14\% after 24 hours $(P<0.01)$ and $20 \%$ after 48 hours $(P<0.01))$ (Additional file 4: Figure S3B). Following food deprivation, a reduction in muscle weight was found for the gastrocnemius/plantaris muscles (15\% after 48 hours $(P<0.001)$ of starvation), but not tibialis anterior muscle 
A

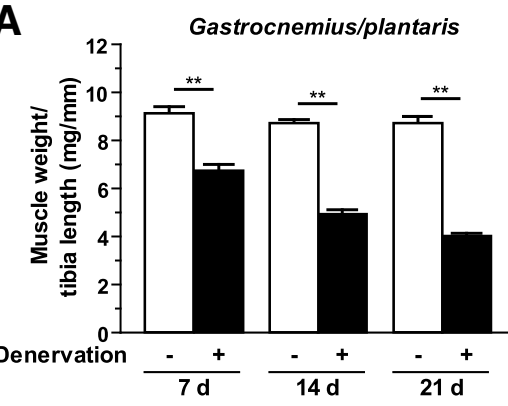

B

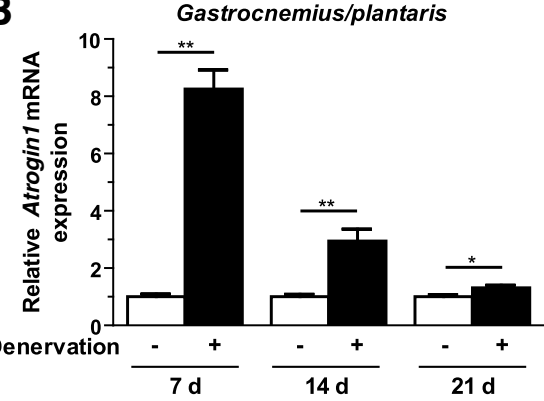

C

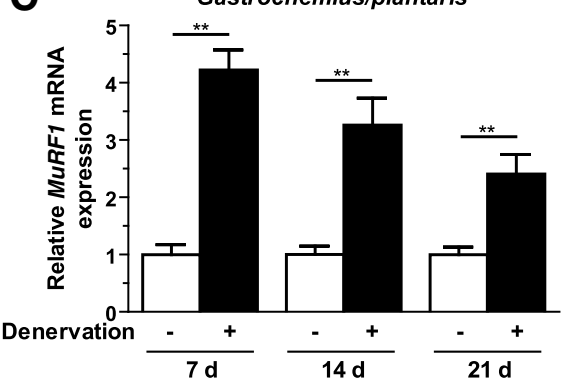

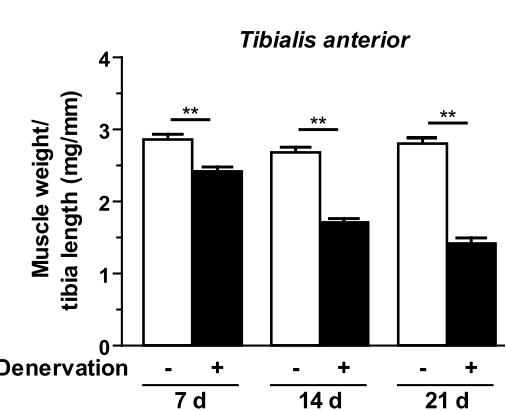

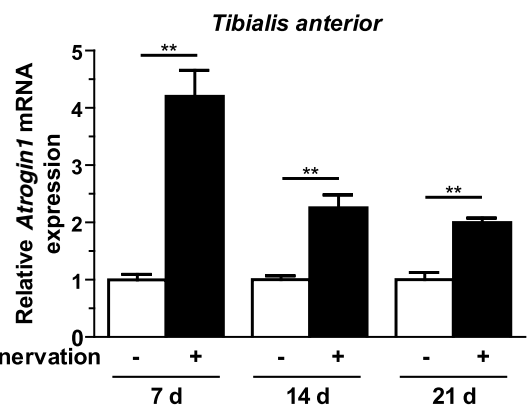

Tibialis anterior

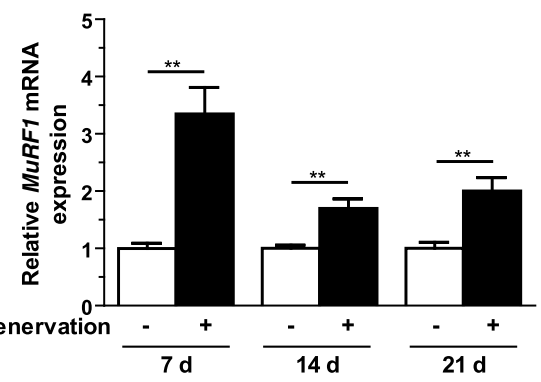

Tibialis anterior
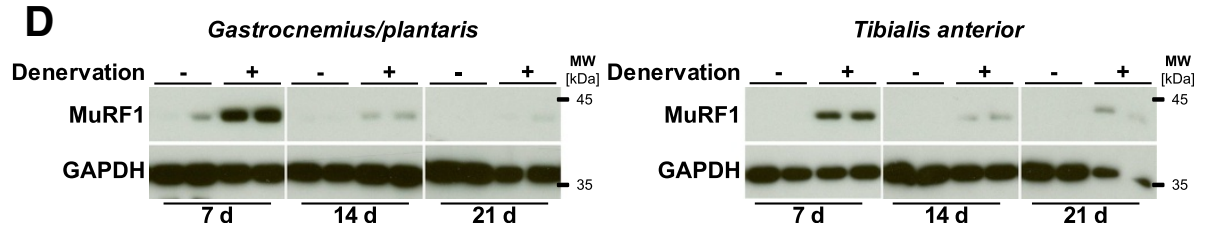

Figure 4 Denervation-induced atrophy. Six- to eight-week-old male C57BL/6 N mice were subjected to surgery. The sciatic nerve of the left hindlimb was dissected (denervated, $n=6$ ), and a sham procedure was performed at the right side (innervated, $n=6$ ), as indicated. (A) The weights of gastrocnemius/plantaris and tibialis anterior muscles normalized to tibia length were determined at baseline (-) and after 7 days, 14 days and 21 days. Data are presented as mean \pm SEM. ${ }^{* *} P<0.05$. Quantitative RT-PCR analyses of Atrogin1 (B) and MuRF1 (C) expression and immunoblotting of proteins using anti-MuRF1 antibody (D) in gastrocnemius/plantaris and tibialis anterior muscles at 7 days, 14 days and 21 days after surgery, as indicated. Glyceraldehyde 3-phosphate dehydrogenase (Gapdh) expression and protein content were used as reference values, and data shown are the fold changes of the respective innervated sites. Data are presented as mean \pm SEM. ${ }^{* *} P<0.01$, ${ }^{*} P<0.05$. MuRF1, Muscle RING (really interesting new gene) finger-containing protein 1.

(Figure 5A). qRT-PCR experiments showed an upregulation of muscular Atrogin1 and MuRF1 expression during starvation (Figures 5B and 5C). Atrogin1 expression increased 21.6-fold and 29.4-fold after 24 hours and 48 hours of starvation, respectively, in gastrocnemius/ plantaris muscles $(P<0.01$ for both). Atrogin1 expression was also increased in tibialis anterior muscle (Figure $5 \mathrm{~B}$ ). In gastrocnemius/plantaris muscles, MuRF1 expression increased 21-fold and 24-fold after 24 hours and 48 hours of starvation, respectively ( $P<0.01$ for both). $M u R F 1$ expression was also increased in tibialis anterior muscle (Figure 5C). The MuRF1 protein content was increased in gastrocnemius/plantaris and tibialis anterior muscles of mice subjected to starvation (Figure 5D).

Fasting resulted in a significant increase in muscular Trim62 expression after 24 hours and 48 hours of 
A

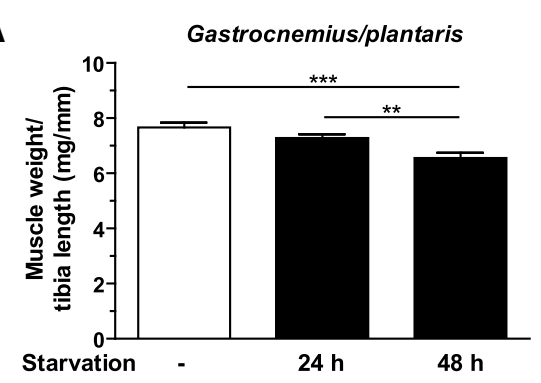

B

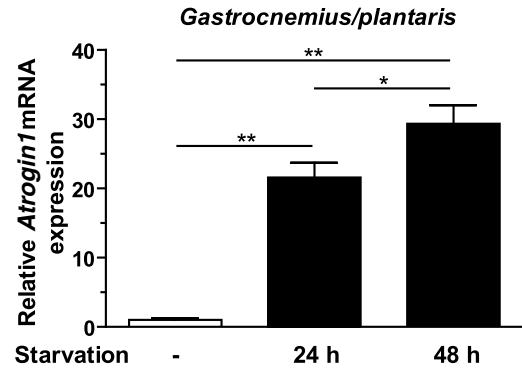

C

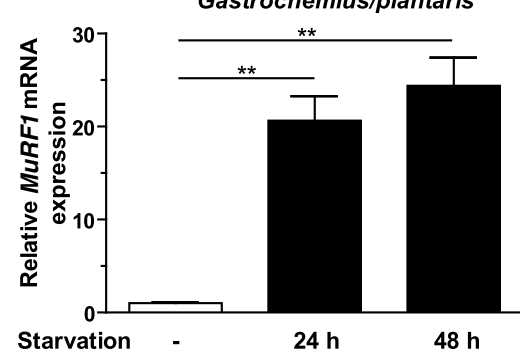

D

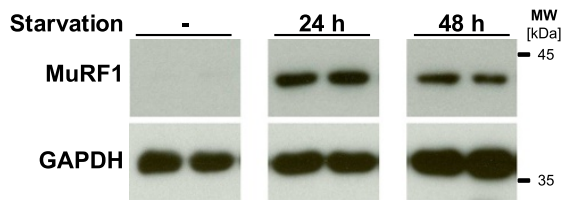

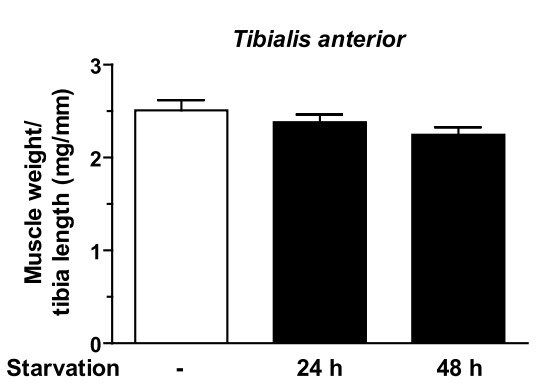
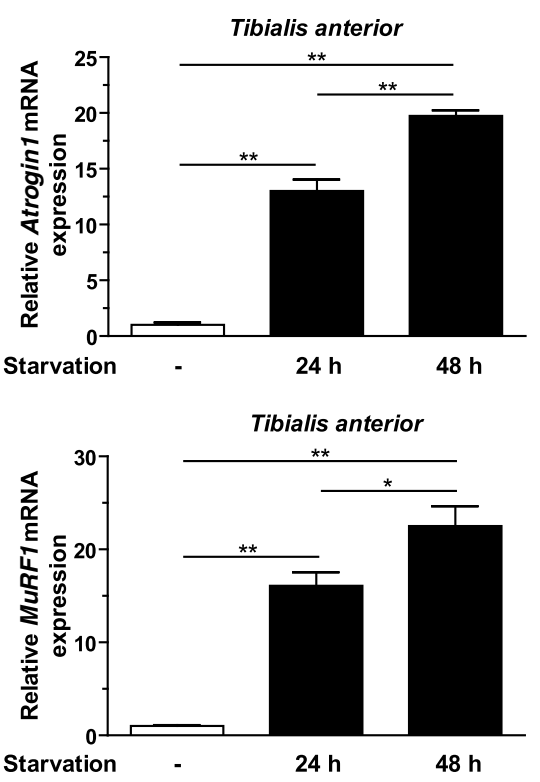

Tibialis anterior

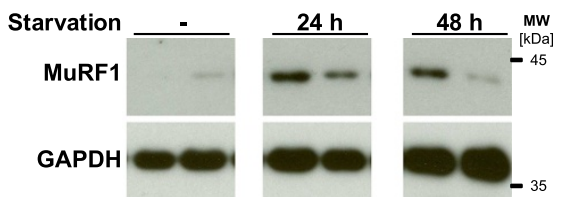

Figure 5 Starvation leads to skeletal muscle atrophy in vivo. Six- to eight-week-old male C57BL/6 N mice were subjected to food deprivation for 24 hours $(n=6)$ or 48 hours $(n=6)$, as indicated. Mice fed standard chow (controls, $-; n=6)$ were used as controls. (A) Weights of gastrocnemius/ plantaris and tibialis anterior muscles normalized to tibia length are shown. Data are presented as mean $\pm \mathrm{SEM}$. ${ }^{* * *} P<0.001$, ${ }^{* *} P<0.01$. Quantitative RT-PCR analyses of Atrogin1 (B) and MURF1 (C) expression, and immunoblotting of proteins using anti-MuRF1 antibody (D), in gastrocnemius/plantaris and tibialis anterior muscles of control animals (-) and mice deprived of food for 24 hours and 48 hours are shown, as indicated. Glyceraldehyde 3-phosphate dehydrogenase (Gapdh) expression and protein content were used as reference values, and data shown are fold changes compared to controls. Data are presented as mean \pm SEM. ${ }^{* *} P<0.01,{ }^{*} P<0.05$. MuRF1, Muscle RING (really interesting new gene) finger-containing protein 1.

starvation (Figure 3C). Trim62 expression increased 1.6fold after 24 hours $(P<0.05)$ and remained at this level at 48 hours $(P<0.05)$ of starvation in gastrocnemius/ plantaris and tibialis anterior muscles $(P<0.01$ for both).

Trim62 contributes to inflammatory response in muscle cells Immunocytochemistry was performed to elucidate whether endogenous Trim62 protein was contained in myocytes. As expected, differentiated $\mathrm{C} 2 \mathrm{C} 12$ myotubes contained Trim62 endogenously. Trim62 was exclusively localized in the cytoplasm of these cells (Figure 6A). The localization of Trim62 in the cytoplasm was confirmed when we transfected expression plasmids encoding Trim62 with either an amino-terminal FLAG tag or a carboxy-terminal myc/His-6 tag in $\mathrm{C} 2 \mathrm{C} 12$ myoblasts (Figure 6B). These data indicate that Trim62 is contained in myocytes, where it is localized mainly in the cytoplasm. 
A
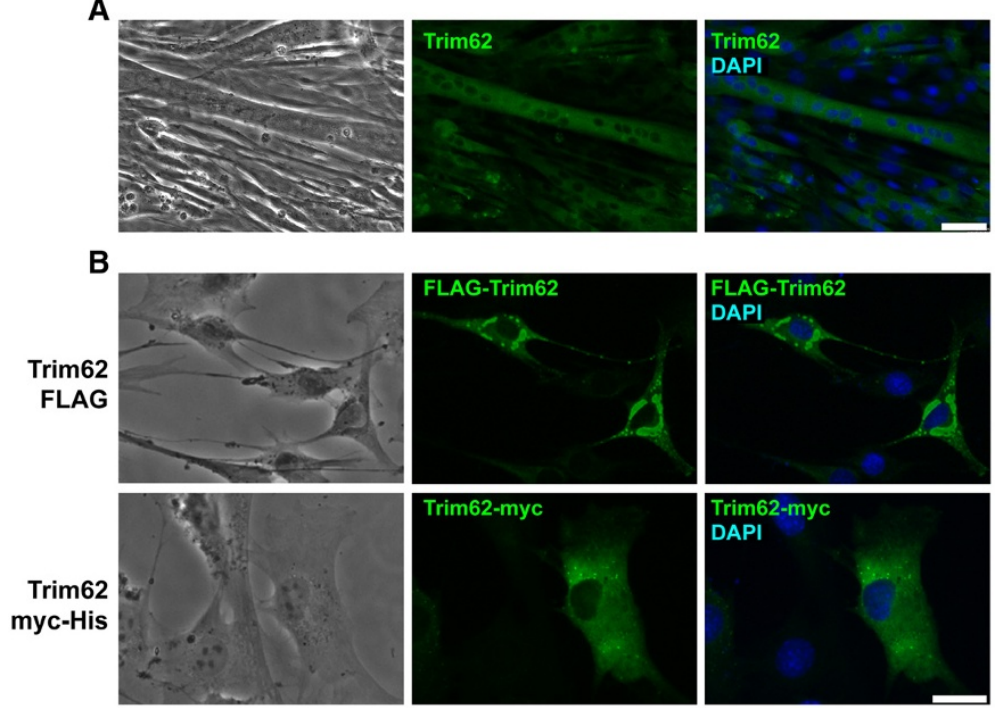

Figure 6 Trim62 is expressed in myocytes. (A) Immunocytochemistry of differentiated C2C12 myotubes was performed using an anti-Trim62 antibody. Nuclei were stained with 4,6-diamidino-2-phenylindole (DAPI, blue). Scale bar, 50 um. (B) C2C12 myoblasts were transfected with expression plasmids encoding Trim62-FLAG (top panel), Trim62 myc/His-6 (bottom panel) or an empty vector control plasmid. Immunofluorescence analysis was performed using anti-FLAG and anti-Myc antibodies, respectively, as well as an Alexa Fluor 488-coupled secondary antibody. Nuclei were stained with DAPI (blue). Scale bar, $25 \mu \mathrm{m}$.

Trim62 was implicated in the inflammatory response of immune cells by regulating the TLR4 signaling pathway, leading to activation of AP-1 [21]. Therefore, we analyzed whether overexpressed Trim62 affects a reporter construct harboring three consecutive AP-1 consensus sites. Indeed, overexpression of Trim62 induced the AP-1dependent promoter construct, indicating that Trim62 activates AP-1-dependent signaling events (Figure 7A). We also tested whether deletion of Trim62 in differentiated $\mathrm{C} 2 \mathrm{C} 12$ myotubes by siRNA has an effect on TLR4mediated activation of AP-1. Following knockdown of Trim62 in $\mathrm{C} 2 \mathrm{C} 12$ myotubes (Figure 7B), we treated these cells with the TLR4 agonist lipopolysaccharide (LPS) and analyzed Il-6 expression by qRT-PCR. As expected, LPS treatment led to increased $\mathrm{Il}-6$ expression in myocytes (Figure 7C). When Trim62 was downregulated, the LPS-mediated increase in $\mathrm{Il}-6$ expression was diminished. These data indicate that Trim62 is involved in LPS-induced Il-6 expression and thus in the inflammatory response in myocytes.

\section{Discussion}

Increased TRIM62 gene expression and protein content were found in skeletal muscle of critically ill patients. This finding was confirmed by increased Trim62 expression in skeletal muscle of three muscle atrophy mouse models. In contrast to decreasing levels of Atrogin1 and MuRF1 during the late phase of muscle atrophy, Trim62 levels remained continuously elevated in all atrophy models. These data implicate a role of Trim62 for the later phase of muscle atrophy. Because Trim62 activates the TRIF branch of TLR4 signaling which leads to increased LPS-induced $I L-6$ expression [21], we propose that continuous Trim62 activation contributes to persistent inflammation in muscle, promoting atrophy and development of ICUAW in critically ill patients. To our knowledge, the present study is the first in which TRIM62 regulation has been investigated in human and mouse skeletal muscle during atrophy.

TRIM62 belongs to the TRIM family of RING finger E3 ubiquitin ligases $[18,19]$ that is involved in the regulation of differentiation, immunity, development and apoptosis [18]. This protein family is also involved in muscular protein homeostasis. In muscle, TRIM mutations lead to primary myopathies. For instance, mutated TRIM32 leads to limb-girdle muscular dystrophy type $2 \mathrm{H}$ [27]; mutations in TRIM18 (MID1) cause Opitz G/BBB syndrome [28]; and TRIM63/MuRF1 mutations result in hypertrophic cardiomyopathy [29]. The MuRF family (MuRF1/TRIM63, MuRF2/TRIM55 and MuRF3/TRIM54) of TRIM proteins has essential functions for protein homeostasis in striated muscle responsible for myogenesis [30] and maintenance [10,31-33]. Our data indicate that TRIM62 is also involved in muscular protein homeostasis, especially during inflammation-induced atrophy. We have established TRIM62 as novel atrophy marker. However, further studies are needed to understand its importance in muscle atrophy.

Critically ill patients often develop ICUAW as a severe complication of critical illness. It affects more than half 


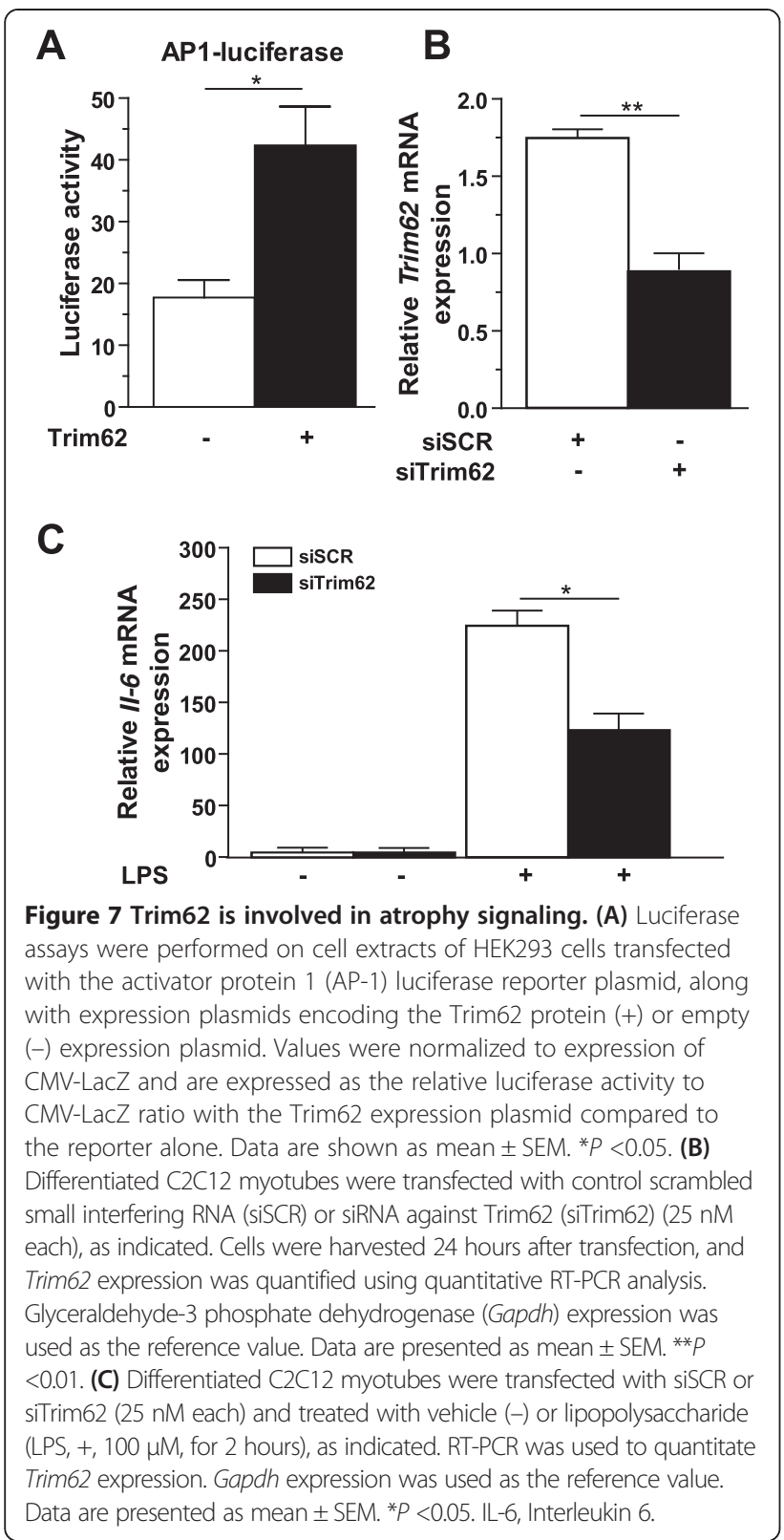

of all ICU patients [34]. ICUAW is characterized by skeletal muscle atrophy and weakness [2-4], is associated with elevated morbidity and mortality and impairs shortand long-term clinical outcomes [6,35]. Recently, we found persistently increased $I L-6$ expression and elevated SAA1 content in muscles of critically ill patients $[13,24]$. On the basis of these data, we hypothesized that continuous inflammation and acute-phase response in muscle play an important role in ICUAW [13,24]. Our observation that TRIM62 is persistently increased in muscles of critically ill patients at high risk of developing ICUAW and in all atrophy mouse models strengthens this hypothesis. Knockdown of TRIM62 in primary macrophages abolished TRIF-mediated AP-1 signal transduction following LPS treatment [21]. We show that this pathway is also active in myocytes in our finding that knockdown of Trim62 inhibited LPS-induced Il-6 expression in $\mathrm{C} 2 \mathrm{C} 12$ cells. These proinflammatory actions of TRIM62 might explain our recent finding of persistently increased $I L-6$ expression in muscles of ICUAW patients $[13,24]$. Because AP-1 signaling is essential in denervation-induced atrophy [22], TRIM62mediated AP-1 activation is particularly important. We confirm that Trim62 is involved in this cascade, because its overexpression increased AP-1 activity in myocytes. We propose that persistent activation of TRIM62 in muscle could lead to chronically increased AP-1 activity, promoting atrophy even when Atrogin1 and MuRF1 expression have normalized.

An imbalanced muscular protein homeostasis with increased UPS-mediated protein degradation and reduced protein synthesis plays a dominant role in critically ill patients $[1,8]$. Atrogin 1 and MuRF1 are key factors in this pathway and are consistently used as atrophy markers [12]. Atrogin1 and MuRF1 are rapidly, but only transiently, increased in muscles of critically ill patients [8] and in atrophy mouse models [12]. This time course of Atrogin1 and MuRF1 expression is consistent with our findings reported here. In contrast, muscular Trim62 expression remained increased throughout the disease course in ICUAW patients and in all mouse models. These findings support the assumption that Atrogin1 and MuRF1 are predominantly involved in the early phase of atrophy. We suggest that early and sustained muscular Trim62 expression continuously activate atrophy signaling pathways involved in later phases of the disease process. Increased muscular Trim62 expression in all atrophy models implicates that Trim62 upregulation is a general and nonspecific feature in muscle atrophy.

\section{Limitations}

We used CLP to investigate inflammation-induced muscle atrophy. This method causes polymicrobial sepsis and systemic inflammation in mice. Therefore, we cannot define a precise pathomechanism (that is, specific inflammatory cytokines, bacteria or fungi) responsible for inflammation-induced muscle atrophy. However, CLP is considered a gold standard in sepsis research $[25,36,37]$. The pathomechanisms and cytokine release following CLP are close to the human situation in sepsis [38-40]. Further studies are needed to investigate specific pathways in inflammation-induced atrophy.

The balance between protein synthesis and degradation responsible for maintenance of muscular protein homeostasis is disturbed in critically ill patients [1]. In our present study, we found an increased atrogene gene expression indicative for enhanced UPS-dependent protein degradation and muscle atrophy [12,41,42]. However, we did not 
investigate whether protein synthesis was reduced in our models. Therefore, we cannot draw any conclusions about whether muscle atrophy occurred because of increased protein degradation alone or whether an accompanying decrease in protein synthesis contributed to this phenotype.

\section{Conclusions}

Chronic inflammation and acute-phase response in muscle plays an important role in ICUAW pathogenesis $[13,24]$. TRIM62 might be a new factor responsible for persistent inflammation in muscle that promotes muscle atrophy and ICUAW. TRIM62 gene expression and protein content were increased in muscles of critically ill patients and three standard murine muscle atrophy models. Continuously increased muscular TRIM62 expression throughout the disease course in ICUAW patients and in all mouse models was in clear contrast to the time course of the commonly used atrophy markers Atrogin1 and MuRF1, which are rapidly, but only transiently, increased in muscles of critically ill patients [8] and murine muscle atrophy models [12]. Sustained muscular TRIM62 expression might point toward its role in later phases of muscle atrophy, whereas Atrogin1 and MuRF1 are involved predominantly in the early disease phase. With these data, we establish TRIM62 as a novel marker for muscle atrophy. In summary, we think that Trim62 contributes to inflammation-induced muscle atrophy at least in part by activation of the AP-1 signal transduction pathway.

\section{Key messages}

- Persistent inflammation and acute-phase response in muscle contributes to the pathogenesis of ICUAW.

- The E3 ubiquitin ligase and activator of inflammation TRIM62 is continuously increased in skeletal muscle of ICUAW patients and mouse models of inflammation-induced atrophy.

- In contrast to bona fide atrogenes, TRIM62 expression remains increased in atrophic muscle throughout the disease process.

- Overexpression of Trim62 activated the atrophyinducing AP-1 signal transduction pathway in myocytes, and its knockdown inhibited LPS-induced $\mathrm{Il}-6$ expression.

- TRIM62 could play a role in ICUAW pathogenesis by activating and maintaining inflammation in muscle.

\section{Additional files}

Additional file 1: Table S1. Patient characteristics. ALI/ARDS, Acute lung injury/acute respiratory distress syndrome; BMI, Body mass index; CNS, Central nervous system; ICU, Intensive care unit; MRC, Medical Research Council; NA, Not applicable; ND, Not determined; SOFA,
Sequential Organ Failure Assessment score; SAPS-II, Simplified Acute Physiology Score II. Table S2. Treatment of ICU patients during study period. RASS, Richmond Agitation Sedation Scale; PBW, Predicted body weight. Data shown are median (IQR) or number and percentage. Table S3. Primer pairs for quantitative RT-PCR are shown. TRIM62, Tripartite motif-containing 62; Saa1, Serum amyloid A1; MuRF1, Muscle RING (really interesting new gene) finger-containing protein; GAPDH, Glyceraldehyde 3-phosphate dehydrogenase; IL-6, Interleukin 6; Tnfa, Tumor necrosis factor a; Hs, Homo sapiens; Mm, Mus musculus.

Additional file 2: Figure S1. Study protocol.

Additional file 3: Figure S2. CLP-induced inflammation and acute-phase response in liver. Quantitative RT-PCR analyses of interleukin 6 (IL-6), tumor necrosis factor a (Tnfa) and serum amyloid A1 (Saa 1) expression in the liver 24 hours, 48 hours, 72 hours or 96 hours after surgery, as indicated. Glyceraldehyde 3-phosphate dehydrogenase expression was used as a reference, and data are shown as relative expression. Data presented are mean \pm SEM. ${ }^{* *} P<0.01,{ }^{*} P<0.05$.

Additional file 4: Figure S3. Body weight during skeletal muscle atrophy. Body weight normalized to tibia length is shown for skeletal muscle atrophy induced by CLP (A), starvation (B) and denervation (C). Data presented are mean \pm SEM. ${ }^{* *} P<0.01,{ }^{*} P<0.05$.

\section{Abbreviations}

AP-1: Activator protein 1; CLP: Cecal ligation and puncture; ICUAW: ICU-acquired weakness; II-6: Interleukin 6; MuRF: Muscle RING (really interesting new gene) finger-containing protein; MyHC: Myosin heavy chain; qRT-PCR: Quantitative real-time polymerase chain reaction; SAA: Serum amyloid A; TLR4: Toll-like receptor 4; TRIF: Toll/interleukin 1 receptor domain-containing adapter inducing interferon $\beta$; Trim: Tripartite motif-containing.

\section{Competing interests}

The authors declare that they have no competing interests.

\section{Authors' contributions}

FS, MK, XZ, CL, DL and JF designed and analyzed the experiments and prepared the manuscript. KP performed and analyzed the experiments. TW, CK and SWC conducted and analyzed the clinical trial. All authors read and approved the final manuscript.

\section{Acknowledgements}

We thank Friedrich C Luft for assistance and advice. We thank Janine Woehlecke and Sibylle Schmidt for excellent technical assistance. We thank Simone Spuler for sharing patient samples and Siegfried Labeit for sharing the anti-MuRF1 antibody. We are grateful for the patience and courage of our patients and their consenting relatives. This work was supported by the Deutsche Forschungsgemeinschaft (FI 965/4-1 KFO 192 and FI 965/5-1), a Marie Curie International Reintegration grant and MyoGrad (Deutsche Forschungsgemeinschaft, GK1631).

\section{Author details}

${ }^{1}$ Experimental and Clinical Research Center (ECRC), a Cooperation between Max-Delbrück-Centrum and Charité-Universitätsmedizin Berlin, Campus Buch, Lindenberger Weg 80, 13125 Berlin, Germany. ${ }^{2}$ Charité-Universitätsmedizin Berlin, Campus Virchow and Campus Mitte, Anesthesiology and Operative Intensive Care Medicine, Augustenburger Platz 1, 13353 Berlin, Germany. ${ }^{3}$ Charité-Universitätsmedizin Berlin, Campus Virchow, Center for Musculoskeletal Surgery, Augustenburger Platz 1, 13353 Berlin, Germany. ${ }^{4}$ Cardiology, Charité-Universitätsmedizin Berlin, Campus Virchow,

Augustenburger Platz 1, 13353 Berlin, Germany.

Received: 5 May 2014 Accepted: 11 September 2014 Published online: 29 September 2014

\section{References}

1. Puthucheary ZA, Rawal J, McPhail M, Connolly B, Ratnayake G, Chan P, Hopkinson NS, Padhke R, Dew T, Sidhu PS, Velloso C, Seymour J, Agley CC, Selby A, Limb M, Edwards LM, Smith K, Rowlerson A, Rennie MJ, Moxham J, Harridge SD, Hart N, Montgomery HE: Acute skeletal muscle wasting in critical illness. JAMA 2013, 310:1591-1600. 
2. Bierbrauer J, Koch S, Olbricht C, Hamati J, Lodka D, Schneider J, Luther-Schroder A, Kleber C, Faust K, Wiesener S, Spies CD, Spranger J, Spuler S, Fielitz J, Weber-Carstens S: Early type II fiber atrophy in intensive care unit patients with nonexcitable muscle membrane. Crit Care Med 2012, 40:647-650.

3. Lefaucheur JP, Nordine T, Rodriguez P, Brochard L: Origin of ICU acquired paresis determined by direct muscle stimulation. I Neurol Neurosurg Psychiatry 2006, 77:500-506.

4. Bolton CF, Young GB, Zochodne DW: The neurological complications of sepsis. Ann Neurol 1993, 33:94-100.

5. De Jonghe B, Sharshar T, Lefaucheur JP, Authier FJ, Durand-Zaleski I, Boussarsar M, Cerf C, Renaud E, Mesrati F, Carlet J, Raphaël JC, Outin H, Bastuji-Garin S, the Groupe de Réflexion et d'Etude des Neuromyopathies en Réanimation: Paresis acquired in the intensive care unit: a prospective multicenter study. JAMA 2002, 288:2859-2867.

6. Herridge MS, Tansey CM, Matté A, Tomlinson G, Diaz-Granados N, Cooper A, Guest CB, Mazer CD, Mehta S, Stewart TE, Kudlow P, Cook D, Slutsky AS, Cheung AM, the Canadian Critical Care Trials Group: Functional disability 5 years after acute respiratory distress syndrome. N Engl J Med 2011, 364:1293-1304.

7. Friedrich O: Critical illness myopathy: What is happening? Curr Opin Clin Nutr Metab Care 2006, 9:403-409.

8. Wollersheim T, Woehlecke J, Krebs M, Hamati J, Lodka D, Luther-Schroeder A, Langhans C, Haas K, Radtke T, Kleber C, Spies C, Labeit S, Schuelke M, Spuler S, Spranger J, Weber-Carstens S, Fielitz J: Dynamics of myosin degradation in intensive care unit-acquired weakness during severe critical illness. Intensive Care Med 2014, 40:528-538.

9. Klaude M, Mori M, Tjäder I, Gustafsson T, Wernerman J, Rooyackers O: Protein metabolism and gene expression in skeletal muscle of critically ill patients with sepsis. Clin Sci 2012, 122:133-142.

10. Fielitz J, Kim MS, Shelton JM, Latif S, Spencer JA, Glass DJ, Richardson JA, Bassel-Duby R, Olson EN: Myosin accumulation and striated muscle myopathy result from the loss of muscle RING finger 1 and 3. J Clin Invest 2007, 117:2486-2495.

11. Constantin D, McCullough J, Mahajan RP, Greenhaff PL: Novel events in the molecular regulation of muscle mass in critically ill patients. J Physiol 2011, 589:3883-3895.

12. Bodine SC, Latres E, Baumhueter S, Lai VK, Nunez L, Clarke BA, Poueymirou WT, Panaro FJ, Na E, Dharmarajan K, Pan ZQ, Valenzuela DM, DeChiara TM, Stitt TN, Yancopoulos GD, Glass DJ: Identification of ubiquitin ligases required for skeletal muscle atrophy. Science 2001, 294:1704-1708.

13. Langhans C, Weber-Carstens S, Schmidt F, Hamati J, Kny M, Zhu X, Wollersheim T, Koch S, Krebs M, Schulz H, Lodka D, Saar K, Labeit S, Spies C, Hubner N, Spranger J, Spuler S, Boschmann M, Dittmar G, Butler-Browne G, Mouly V, Fielitz J: Inflammation-induced acute phase response in skeletal muscle and critical illness myopathy. PLoS One 2014, 9:e92048.

14. Tsujinaka T, Fujita J, Ebisui C, Yano M, Kominami E, Suzuki K, Tanaka K, Katsume A, Ohsugi Y, Shiozaki H, Monden M: Interleukin 6 receptor antibody inhibits muscle atrophy and modulates proteolytic systems in interleukin 6 transgenic mice. J Clin Invest 1996, 97:244-249.

15. Zimmers TA, McKillop $\mathrm{H}_{\text {, }}$ Pierce RH, Yoo JY, Koniaris LG: Massive liver growth in mice induced by systemic interleukin 6 administration. Hepatology 2003, 38:326-334.

16. Zamir O, Hasselgren PO, Kunkel SL, Frederick J, Higashiguchi T, Fischer JE: Evidence that tumor necrosis factor participates in the regulation of muscle proteolysis during sepsis. Arch Surg 1992, 127:170-174.

17. Goodman MN: Tumor necrosis factor induces skeletal muscle protein breakdown in rats. Am J Physiol Endocrinol Metab 1991, 260:E727-E730.

18. Reymond A, Meroni G, Fantozzi A, Merla G, Cairo S, Luzi L, Riganelli D, Zanaria E, Messali S, Cainarca S, Guffanti A, Minucci S, Pelicci PG, Ballabio A: The tripartite motif family identifies cell compartments. EMBO J 2001, 20:2140-2151.

19. McNab FW, Rajsbaum R, Stoye JP, O'Garra A: Tripartite-motif proteins and innate immune regulation. Curr Opin Immunol 2011, 23:46-56.

20. Lott ST, Chen N, Chandler DS, Yang Q, Wang L, Rodriguez M, Xie H, Balasenthil S, Buchholz TA, Sahin AA, Chaung K, Zhang B, Olufemi SE, Chen J, Adams H, Band V, El-Naggar AK, Frazier ML, Keyomarsi K, Hunt KK, Sen S, Haffty B, Hewitt SM, Krahe R, Killary AM: DEAR1 is a dominant regulator of acinar morphogenesis and an independent predictor of local recurrence-free survival in early-onset breast cancer. PLOS Med 2009, 6:e1000068.
21. Uchil PD, Hinz A, Siegel S, Coenen-Stass A, Pertel T, Luban J, Mothes W: TRIM protein-mediated regulation of inflammatory and innate immune signaling and its association with antiretroviral activity. J Virol 2013, 87:257-272.

22. Choi MC, Cohen TJ, Barrientos T, Wang B, Li M, Simmons BJ, Yang JS, Cox GA, Zhao Y, Yao TP: A direct HDAC4-MAP kinase crosstalk activates muscle atrophy program. Mol Cell 2012, 47:122-132.

23. Weber-Carstens S, Schneider J, Wollersheim T, Assmann A, Bierbrauer J, Marg A, Al Hasani H, Chadt A, Wenzel K, Koch S, Fielitz J, Kleber C, Faust K, Mai K, Spies CD, Luft FC, Boschmann M, Spranger J, Spuler S: Critical illness myopathy and GLUT4: significance of insulin and muscle contraction. Am J Respir Crit Care Med 2013, 187:387-396.

24. Weber-Carstens S, Deja M, Koch S, Spranger J, Bubser F, Wernecke KD, Spies CD, Spuler S, Keh D: Risk factors in critical illness myopathy during the early course of critical illness: a prospective observational study. Crit Care 2010, 14:R119.

25. Rittirsch D, Huber-Lang MS, Flierl MA, Ward PA: Immunodesign of experimental sepsis by cecal ligation and puncture. Nat Protoc 2009, 4:31-36.

26. Kim MS, Fielitz J, McAnally J, Shelton JM, Lemon DD, McKinsey TA Richardson JA, Bassel-Duby R, Olson EN: Protein kinase D1 stimulates MEF2 activity in skeletal muscle and enhances muscle performance. Mol Cell Biol 2008, 28:3600-3609.

27. Frosk P, Weiler T, Nylen E, Sudha T, Greenberg CR, Morgan K, Fujiwara TM, Wrogemann $\mathrm{K}$ : Limb-girdle muscular dystrophy type $2 \mathrm{H}$ associated with mutation in TRIM32, a putative E3-ubiquitin-ligase gene. Am J Hum Genet 2002, 70:663-672.

28. Quaderi NA, Schweiger S, Gaudenz K, Franco B, Rugarli El, Berger W, Feldman GJ, Volta M, Andolfi G, Gilgenkrantz S, Marion RW, Hennekam RCM, Opitz JM, Muenke M, Ropers HH, Ballabio A: Opitz G/BBB syndrome, a defect of midline development, is due to mutations in a new RING finger gene on Xp22. Nat Genet 1997, 17:285-291.

29. Chen SN, Czernuszewicz G, Tan Y, Lombardi R, Jin J, Willerson JT, Marian AJ: Human molecular genetic and functional studies identify TRIM63, encoding Muscle RING Finger Protein 1, as a novel gene for human hypertrophic cardiomyopathy. Circ Res 2012, 111:907-919.

30. Pizon V, lakovenko A, van der Ven PF, Kelly R, Fatu C, Fürst DO, Karsenti E, Gautel M: Transient association of titin and myosin with microtubules in nascent myofibrils directed by the MURF2 RING-finger protein. J Cell Sci 2002, 115:4469-4482.

31. Fielitz J, van Rooij E, Spencer JA, Shelton JM, Latif S, van der Nagel R, Bezprozvannaya S, de Windt L, Richardson JA, Bassel-Duby R, Olson EN: Loss of muscle-specific RING-finger 3 predisposes the heart to cardiac rupture after myocardial infarction. Proc Natl Acad Sci U S A 2007, 104:4377-4382.

32. Witt CC, Witt SH, Lerche S, Labeit D, Back W, Labeit S: Cooperative control of striated muscle mass and metabolism by MuRF1 and MuRF2. EMBO 2008, 27:350-360.

33. Willis MS, Wadosky KM, Rodriguez JE, Schisler JC, Lockyer P, Hilliard EG, Glass DJ, Patterson C: Muscle ring finger 1 and muscle ring finger 2 are necessary but functionally redundant during developmental cardiac growth and regulate E2F1-mediated gene expression in vivo. Cell Biochem Funct 2014, 32:39-50.

34. Vincent $J$, Norrenberg M: Intensive care unit-acquired weakness: framing the topic. Crit Care Med 2009, 37:S296-\$298.

35. Herridge MS, Cheung AM, Tansey CM, Matte-Martyn A, Diaz-Granados N, AlSaidi F, Cooper AB, Guest CB, Mazer CD, Mehta S, Stewart TE, Barr A, Cook $D$, Slutsky AS, the Canadian Critical Care Trials Group: One-year outcomes in survivors of the acute respiratory distress syndrome. N Engl J Med 2003, 348:683-693.

36. Rittirsch D, Hoesel LM, Ward PA: The disconnect between animal models of sepsis and human sepsis. J Leukoc Biol 2007, 81:137-143.

37. Buras JA, Holzmann B, Sitkovsky M: Animal models of sepsis: setting the stage. Nat Rev Drug Discov 2005, 4:854-865.

38. Remick DG, Newcomb DE, Bolgos GL, Call DR: Comparison of the mortality and inflammatory response of two models of sepsis: lipopolysaccharide vs. cecal ligation and puncture. Shock 2000, 13:110-116.

39. Cavaillon JM, Adib-Conquy M, Fitting C, Adrie C, Payen D: Cytokine cascade in sepsis. Scand J Infect Dis 2003, 35:535-544.

40. Riedemann NC, Guo RF, Ward PA: The enigma of sepsis. J Clin Invest 2003, $112: 460-467$. 
41. Lecker SH, Jagoe RT, Gilbert A, Gomes M, Baracos V, Bailey J, Price SR, Mitch WE, Goldberg AL: Multiple types of skeletal muscle atrophy involve a common program of changes in gene expression. FASEB $J$ 2004, 18:39-51.

42. Lecker SH, Solomon V, Mitch WE, Goldberg AL: Muscle protein breakdown and the critical role of the ubiquitin-proteasome pathway in normal and disease states. J Nutr 1999, 129:227S-237S.

doi:10.1186/s13054-014-0545-6

Cite this article as: Schmidt et al.: The E3 ubiquitin ligase TRIM62 and

inflammation-induced skeletal muscle atrophy. Critical Care 2014 18:545.

\section{Submit your next manuscript to BioMed Central and take full advantage of:}

- Convenient online submission

- Thorough peer review

- No space constraints or color figure charges

- Immediate publication on acceptance

- Inclusion in PubMed, CAS, Scopus and Google Scholar

- Research which is freely available for redistribution 